\title{
Coaxial Electrospinning with Mixed Solvents: From Flat to Round Eudragit L100 Nanofibers for Better Colon-Targeted Sustained Drug Release Profiles
}

\author{
Deng-Guang Yu, ${ }^{1}$ Ying Xu, ${ }^{1,2}$ Zan Li, ${ }^{1}$ Lin-Ping Du, ${ }^{1}$ Ben-Guo Zhao, ${ }^{1}$ and Xia Wang ${ }^{1}$ \\ ${ }^{1}$ School of Materials Science \& Engineering, University of Shanghai for Science and Technology, 516 Jungong Road, \\ Yangpu District, Shanghai 200093, China \\ ${ }^{2}$ School of Environment and Architecture, University of Shanghai for Science and Technology, Shanghai 200093, China \\ Correspondence should be addressed to Deng-Guang Yu; ydg017@usst.edu.cn and Xia Wang; wangxia@usst.edu.cn
}

Received 6 December 2013; Revised 26 January 2014; Accepted 4 March 2014; Published 27 March 2014

Academic Editor: Aihua He

Copyright (C) 2014 Deng-Guang Yu et al. This is an open access article distributed under the Creative Commons Attribution License, which permits unrestricted use, distribution, and reproduction in any medium, provided the original work is properly cited.

\begin{abstract}
A modified coaxial electrospinning process was developed for creating drug-loaded composite nanofibers. Using a mixed solvent of ethanol and N,N-dimethylacetamide as a sheath fluid, the electrospinning of a codissolving solution of diclofenac sodium (DS) and Eudragit L100 (EL100) could run smoothly and continuously without any clogging. A series of analyses were undertaken to characterize the resultant nanofibers from both the modified coaxial process and a one-fluid electrospinning in terms of their morphology, physical form of the components, and their functional performance. Compared with those from the one-fluid electrospinning, the DS-loaded EL100 fibers from the modified coaxial process were rounder and smoother and possessed higher quality in terms of diameter and distribution with the DS existing in the EL100 matrix in an amorphous state; they also provided a better colon-targeted sustained drug release profile with a longer release time period. The modified coaxial process not only can smooth the electrospinning process to prevent clogging of spinneret, but also is a useful tool to tailor the shape of electrospun nanofibers and thus endow them improved functions.
\end{abstract}

\section{Introduction}

The physical properties, such as shape, size, mechanical properties, surface texture, and compartmentalization, profoundly impact the function of a nanobiomaterial and thus raise important questions for the design of its next generation [1]. Electrospinning is a facile method for generating fibers at micro-/nanoscale [2-7]. Often, electrospun nanofibers of some typical pharmaceutical polymers (such as cellulose acetate, Eudragit series, and zein) exhibit a flat morphology, which is undesired for providing sustained drug release profiles because the flat morphology aggravates the initial burst release effect and results in a shorter time sustained release [8-10]. Although a great effort has been paid to generate novel fiber structure (such as core-shell and side-by-side) and obtain nanofibers with thinner diameters [11, 12], few studies have been reported to manipulate the nanofibers' shape and their functional performance.
Coaxial electrospinning is a power tool for generating core-sheath nanofibers through manipulating two fluids using a concentric spinneret $[13,14]$. Traditionally, the shell solution is critical and the selected sheath fluid should be electrospinnable by itself to facilitate core-shell structure formation, whereas the core fluid can either be electrospinnable or not [15]. Yu et al. have broken this concept by developing a modified coaxial electrospinning that is characterized by the usage of liquids lacking electrospinnability as shell fluids [1618]. Because there are many types of "unspinnable" liquids (including solvents, solutions of little chemical molecules, dilute polymer solutions, suspensions, and emulsions) that can be managed to act as sheath fluids for conducting the modified coaxial process, thus this advanced technology has greatly expanded the capability of the traditional coaxial one in generating a series of new possibilities. Some examples include manipulating the size of self-assembled nanoparticles [16]; preparing ultrafine fibers from concentrated polymer 
solutions thought to be unspinnable previously [17]; improving the quality of nanofibers systematically [18]; and coating drug-loaded nanofibers with a blank release-retarded polymer layer for achieving zero-order drug release profiles $[8,9]$.

Here, different from above-mentioned applications, the modified coaxial electrospinning was exploited as a useful tool for manipulating the shape of resultant nanofibers and improving their functional performance of colon-targeted sustained release. Eudragit L-100 (EL100), a well-known methacrylate-based copolymer developed by the Röhm Company in Germany and a common excipient used in the pharmaceutical field, was exploited as the filament-forming matrix here. It has been widely used for the formulation of different oral dosage forms (e.g., tablet coating, tablet matrix, microspheres, and nanoparticles) for colon-targeted drug delivery [19]. Diclofenac sodium (DS), a nonsteroidal antiinflammatory drug (NSAID), was used as a model drug. It is taken or applied to reduce inflammation and as an analgesic reducing pain in certain conditions, supplied as or contained in medications under a variety of trade names [20].

\section{Experimental}

2.1. Materials. EL100 was supplied by Rohm GmbH \& Co. KG (Darmstadt, Germany). DS was purchased from Hubei Biocause Pharmaceutical Co., Ltd. (Hubei, China). Methylene blue, N,N-dimethylacetamide (DMAc), and anhydrous ethanol were provided by Sinopharm Chemical Reagent Co., Ltd. (Shanghai, China). Water was double distilled just before use. All other chemicals and reagents were of analytical grade.

2.2. Preparation. A codissolving solution of EL100 and DS was prepared and used as the core fluid, which consisted of $15 \%(\mathrm{w} / \mathrm{v})$ EL100 and $2 \%(\mathrm{w} / \mathrm{v})$ DS in a mixture of ethanol : DMAc (with a volume ratio of $9: 1$ ), meaning $11.8 \%$ of DS in the solid products. The sheath mixed solvents contained DMAc and ethanol at a volume ratio of $30: 70$. The prepared nanofibers are referred to as F2. To observe the electrospinning process, $5 \mathrm{ppm}$ methylene blue was added to the core solution.

A homemade concentric spinneret was used to conduct the coaxial electrospinning processes. Two syringe pumps (KDS100 and KDS200, Cole-Parmer, IL, USA) and a highvoltage power supply (ZGF $60 \mathrm{Kv} / 2 \mathrm{~mA}^{-1}$, Shanghai Sute Corp., Shanghai, China) were used for electrospinning, which was performed under ambient conditions $\left(25 \pm 2^{\circ} \mathrm{C} ; 57 \% \pm\right.$ $6 \%$ relative humidity). The coaxial electrospinning processes were recorded using a digital video recorder (PowerShot A490, Canon, Tokyo, Japan). After optimization, the applied voltage was fixed at $15 \mathrm{kV}$ and the fibers were collected on an aluminum foil $20 \mathrm{~cm}$ from the spinneret.

Using the same apparatus of coaxial electrospinning and under the same conditions, a one-fluid electrospinning of the core solutions was implemented through adjusting the flow rate of the sheath fluid to $0 \mathrm{~mL} / \mathrm{h}$. The prepared nanofibers are referred to as F1, which was investigated as a control.

2.3. Characterization. The morphology of the nanofibers was examined using an S-4800 field-emission scanning electron microscope (FESEM, Hitachi, Tokyo, Japan). Prior to examination, the samples were platinum sputter-coated under nitrogen atmosphere to render them electrically conductive. The average fiber diameter was determined by measuring their sizes in FESEM images at more than 100 different places using NIH Image J software (National Institutes of Health, MD, USA). Cross-sections of the fiber mats were prepared by placing them in liquid nitrogen and manually breaking them before platinum coating. The topographies of raw material particles and drug-loaded nanofibers were observed under cross-polarized light using an XP-700 polarized optical microscope (Shanghai Changfang Optical Instrument Co. Ltd., Shanghai, China).

$\mathrm{XRD}$ patterns were obtained over the $2 \theta$ range of $5^{\circ}$ to $60^{\circ}$ on a D/Max-BR diffractometer (RigaKu, Tokyo, Japan) with $\mathrm{Cu} \mathrm{K} \alpha$ radiation at $40 \mathrm{mV}$ and $30 \mathrm{~mA}$. Attenuated total reflectance Fourier transform infrared (ATR-FTIR) analysis was carried out on a Nicolet-Nexus 670 FTIR spectrometer (Nicolet Instrument Corporation, Madison, USA) over the range $500-4000 \mathrm{~cm}^{-1}$ and at a resolution of $2 \mathrm{~cm}^{-1}$.

The in vitro dissolution of drug-loaded nanofibers was performed according to the Chinese Pharmacopoeia (2005 Edn.), a paddle method using a RCZ-8A dissolution apparatus (Tianjin University Radio Factory, China). All experiments were conducted at $37^{\circ} \mathrm{C}$ and $50 \mathrm{rpm}$ in $900 \mathrm{~mL} \mathrm{pH} 1.5$ $\mathrm{HCl}$ solutions for $2 \mathrm{~h}$, followed by $5 \mathrm{~h}$ in $900 \mathrm{~mL}$ of phosphate buffer (PBS, $\mathrm{pH} 6.8,0.1 \mathrm{~mol} / \mathrm{L}$ ). At predetermined time intervals, aliquots of $5.0 \mathrm{~mL}$ were withdrawn for sampling and replaced by an equal volume of the same solutions to maintain a constant volume. After filtration through a membrane $(0.45 \mu \mathrm{m})$, the sample solutions were analyzed at a wavelength of $276 \mathrm{~nm}$ by a UV spectrophotometer (UV-2102PC, Unico Instrument Co. Ltd., Shanghai, China). DS dissolved at specified time periods was plotted as percentage released versus time. All measurements were performed six times, and results were reported as the mean value \pm S.D.

\section{Results and Discussion}

3.1. The Modified Coaxial Electrospinning. A schematic diagram of the modified coaxial electrospinning process is shown in Figure 1(a). The homemade spinneret used in this work has outer and inner diameters of 1.2 and $0.3 \mathrm{~mm}$, respectively, (Figure 1(b)). A typical clogging phenomenon that has taken place in the traditional one-fluid electrospinning is shown in Figure 2(a). The easy evaporation of ethanol made a semisolid substance hang on the spinneret to deteriorate the process. The semisolid "skin" had to be removed frequently and manually to keep the electrospinning process going.

Digital images about the modified coaxial electrospinning are shown in Figures 2(b)-2(d). After some preexperiments, a sheath-to-core flow rate ratio of 0.3 was selected for the preparation of drug-loaded nanofibers F2. Under the selected conditions, a fluid jet trajectory similar to that observed in a single-fluid electrospinning was created. This comprised a straight thinning jet emitted from a compound Taylor cone, followed by a bending and whipping instability region with loops of increasing size (Figure $2(\mathrm{c})$ ). The compound Taylor cone is shown in Figure 2(d), which has clearly 


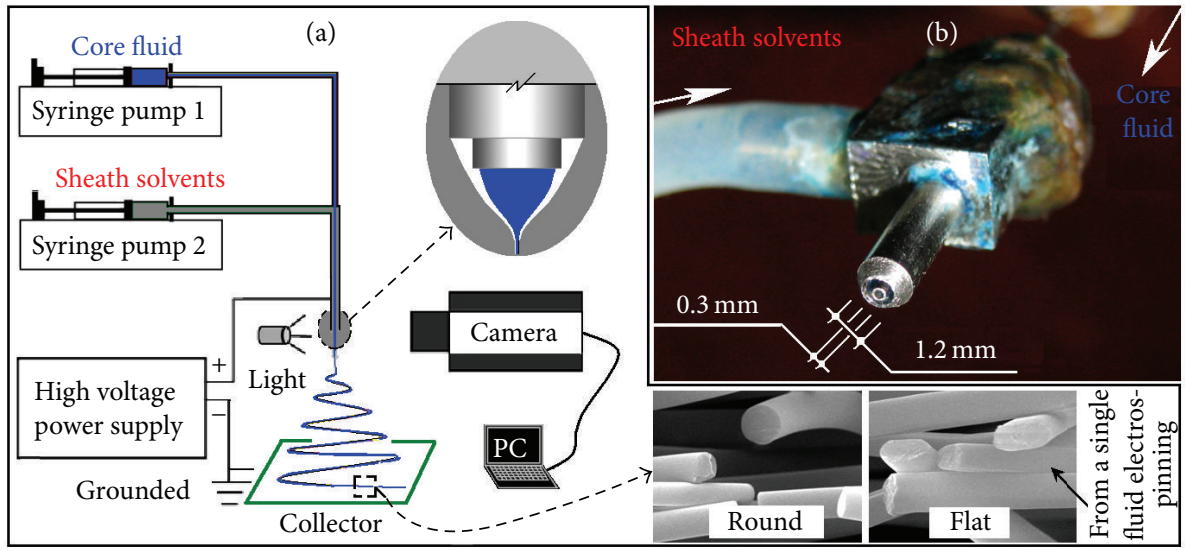

FIGURE 1: The schematic diagram of the modified coaxial electrospinning (a) and the homemade concentric spinneret (b).

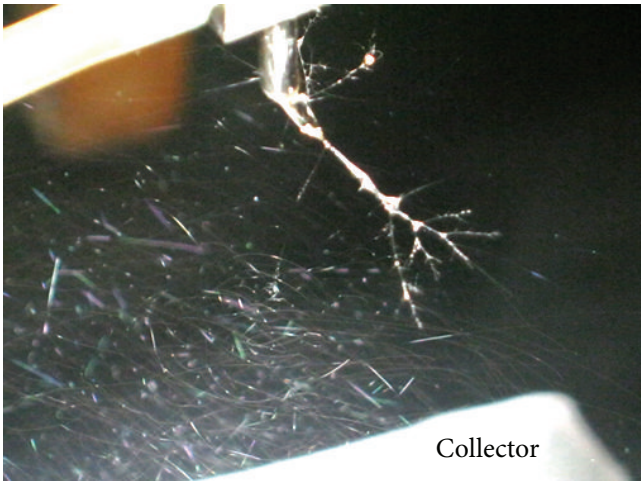

(a)

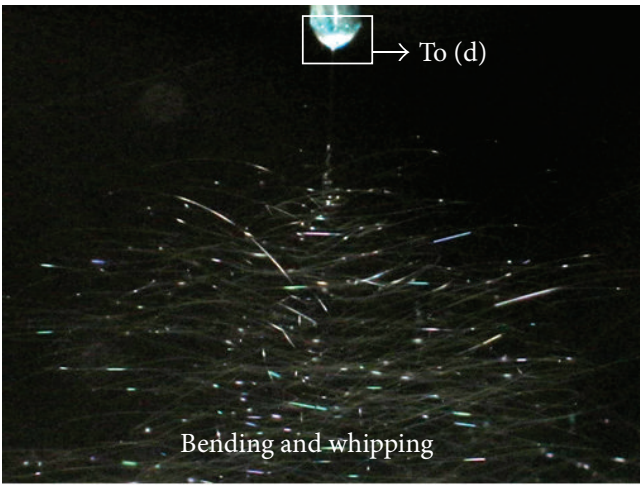

(c)

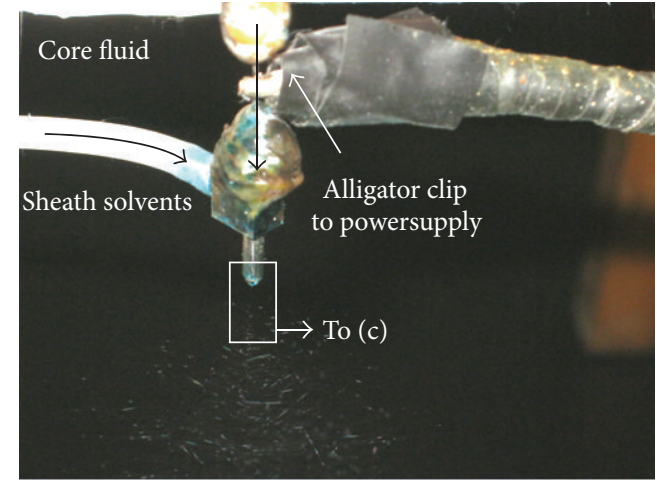

(b)

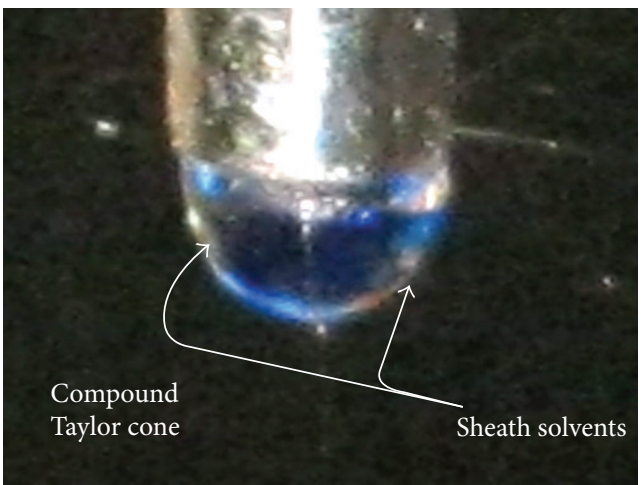

(d)

FIGURE 2: Observations of the traditional single fluid electrospinning (a) and the modified coaxial process ((b) to (d)).

internal and external structure, as indicated by the blue color of methylene blue. The modified coaxial process could be undertaken continuously without any user intervention.

3.2. Morphology. The morphologies of surface (Figures 3(a) and 3(b)) and cross-section (Figures 3(c) and 3(d)) of the nanofibers F1 obtained from the one-fluid electrospinning are given in Figure 3. Clearly, the fibers showed a flat morphology and had an average width of $1280 \pm 330 \mathrm{~nm}$. In sharp contrast, the nanofibers F2 from the modified coaxial processes had rounder and smoother morphologies with a smaller diameter and narrower size distribution of $650 \pm 130 \mathrm{~nm}$, as exhibited by both the surface (Figures $4(\mathrm{a})$ and $4(\mathrm{~b})$ ) and cross-section images (Figures 4(c) and 4(d)).

The traditional one-fluid electrospinning shares characteristics of both electrospraying and conventional solution dry spinning. During the process, ethanol evaporated very quickly. This would make the electrospinning process very sensitive to small changes in the environment and thus resulted in nanofibers with a wide range of sizes and also 



FIGURE 3: Surface and cross-section morphologies of F1 nanofibers from the traditional electrospinning.

generate a solid "skin" on the surfaces of collected fibers with some solvent still trapped inside the fiber bodies. After spinning, the solvent contained in the fibers diffused out into the atmosphere, and the resulting barometric pressure distorted the cylindrical fibers to the flat morphology, whereas the modified coaxial process shares characteristics of both electrospraying and conventional solution wet spinning to some extent. It could provide a stable and robust coresheath interface for the core EL100-DS solution when it was drawn in the electrical field. This not only enables the core solutions to have a longer time period of electrical drawing force in the fluid phase $[16,18]$ but also protects them from the disturbance of environmental changes. During their evaporation, the sheath solvents render a stable Knudsen layer permitting gradual and smooth mass transfer of the solvents from the core fluid jets to the atmosphere and precluding the formation of a surface semisolid "skin" [21]. Thus, the modified process can produce DS-loaded EL100 nanofibers with rounder morphology, smaller diameters, and more uniform structures.
3.3. Physical Status and Compatibility of Components. The presence of numerous distinct peaks in the XRD patterns of the fibers suggested that DS was present as crystalline material with characteristic diffraction peaks, as also demonstrated by the colorful images of their crude particles under polarized light (Figure 5(a)). EL100 exhibits only a hump characteristic of amorphous forms. There are no peaks of crystalline DS detectable in the XRD patterns of the nanofibers F1 and F2, indicating that all the DS encapsulated in the nanofibers no longer presented as a crystalline material regardless of the different electrospinning processes. Unlike the observations on crystalline DS particles, EL100 and the nanofibers showed no bright colors, suggesting that EL100 and the nanofibers were amorphous (Figure 5(a)). These results concurred that DS molecules were highly distributed in the EL100 matrix and were present in a composite manner where the original structure of the pure, crystalline material was lost.

Compared to the spectra of pure DS and EL100, there are significant changes in the spectra of the nanofibers F1 

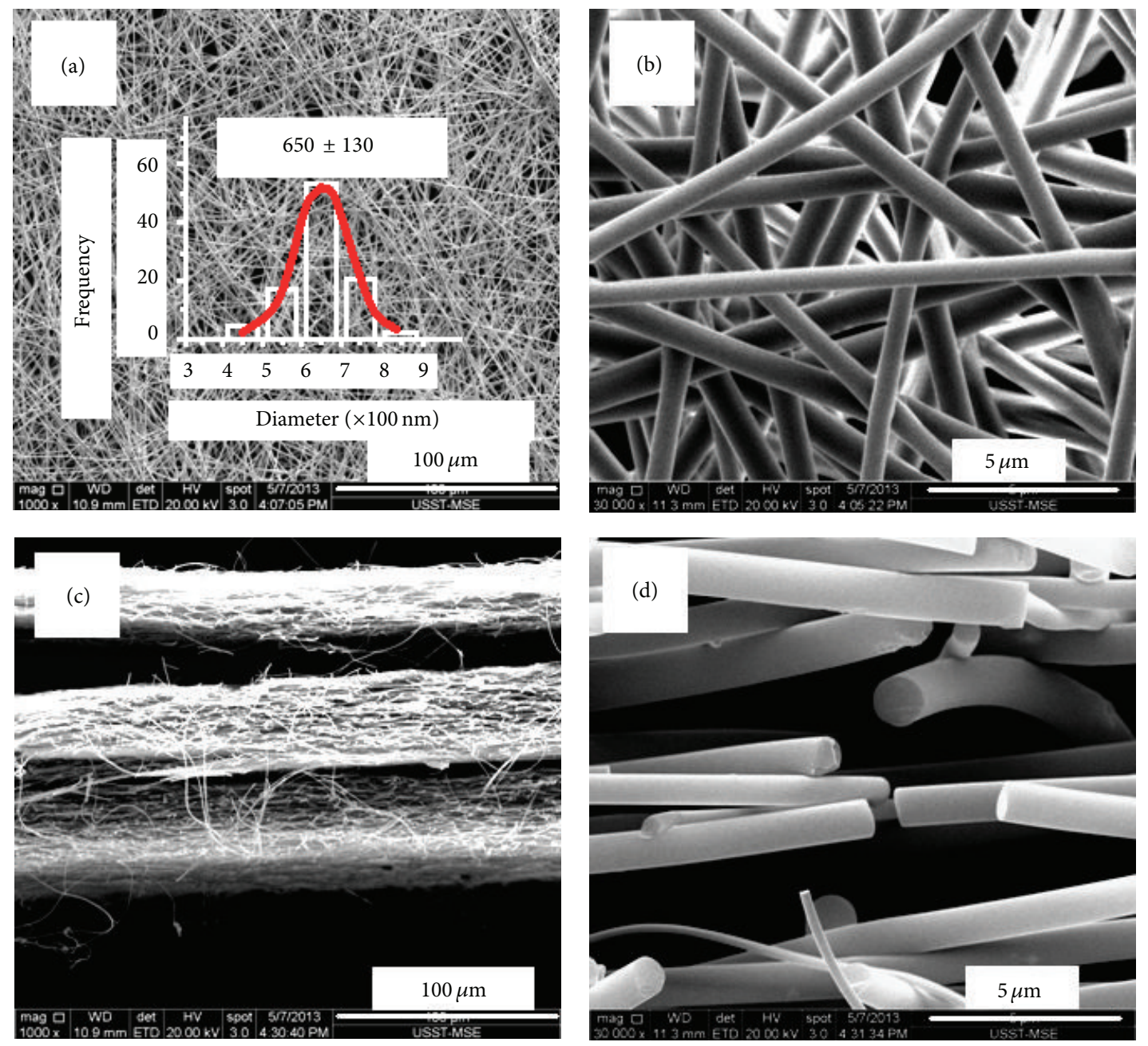

FIGURE 4: Surface and cross-section morphologies of F2 nanofibers from the coaxial process.

and F2 (Figure 5(b)), including (1) the characteristic peaks of the carbonyl groups of EL100 ( $\mathrm{C}=\mathrm{O}$ stretch vibration) at $1735 \mathrm{~cm}^{-1}$ that have shifted to $1732 \mathrm{~cm}^{-1}$; (2) the disappearance of the characteristic peaks of the phenyl group of DS ( $\mathrm{C}=\mathrm{C}$ stretch vibration) at $1577 \mathrm{~cm}^{-1}$ and the disappearance of the substituted phenyl group stretch at $747 \mathrm{~cm}^{-1}$. All these changes can be attributed to hydrogen bonding between DS and EL 100, including (1) between $\mathrm{C}=\mathrm{O}$ of DS and $\mathrm{O}-\mathrm{H}$ of EL 100 and (2) between the C=O of EL 100 and the N-H of DS. The latter hydrogen bonding disrupts the $\mathrm{p}-\pi$ conjugation in the DS molecules generated between the aromatic ring and secondary amino group which has an isolated electron pair at the $\mathrm{N}$ atom and thus accounts for the disappearance of characteristic peaks of DS in the composite nanofibers. The interactions between the DS and EL100 may promote them to combine at a molecular level in the nanofibers, reflecting the good compatibility between them, and would be favorable to the stability of the medicated nanofibers to prevent solid phase separation.

3.4. In Vitro Dissolution Tests. To evaluate DS release profiles from the medicated nanofibers, in vitro dissolution tests were carried out under acidic conditions in $\mathrm{pH} 1.5 \mathrm{HCl}$ for
$2 \mathrm{~h}$ and subsequently at $\mathrm{pH} 6.8$ in $\mathrm{PBS}$ for $5 \mathrm{~h}$ to mimic gastrointestinal conditions. The drug release profiles of the nanofibers are shown in Figure 6(a). In pH $1.5 \mathrm{HCl}$ solutions the in vitro drug release rates were very slow, with no more than $5 \%$ of the loaded drug released from both the samples in $2 \mathrm{~h}$ (Figure 6(a)). Both DS and EL 100 are insoluble in an acid environment; the very small release of drugs from the fibers should reflect the drug content presented on the medicated nanofiber surface.

At a $\mathrm{pH}$ of 6.8 , both the nanofibers $\mathrm{F} 1$ and $\mathrm{F} 2$ exhibited a sustained drug release profile. However, nanofibers F2 from the modified coaxial process provided a better sustained release profile with a longer time period. Compared to nanofibers F2, nanofibers F1 had (1) a larger burst release in the $3 \mathrm{rd}$ hr (i.e., $55.7 \%$ to $41.2 \%$ ) which is disadvantageous to sustained release; (2) a shorter time when $50 \%$ of the contained drug was freed (i.e., $2.8 \mathrm{hr}$ to $3.4 \mathrm{hr}$ ); and (3) a longer time period of the undesired "tailing off" release (i.e., only released $1.9 \%$ after the 5 th $\mathrm{hr}$ ). Both nanofibers $\mathrm{F} 1$ and F2 were nanocomposites with the drug DS homogeneously distributed on the polymer matrix EL100. The sustained drug release profiles should be attributed to their difference in physical shapes, which determined the diffusion or erosion 


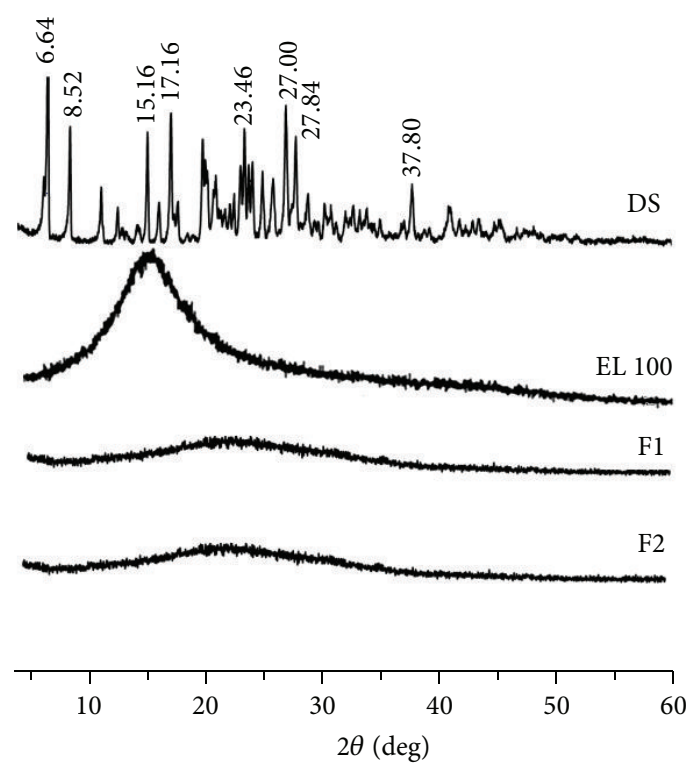

(a)

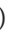

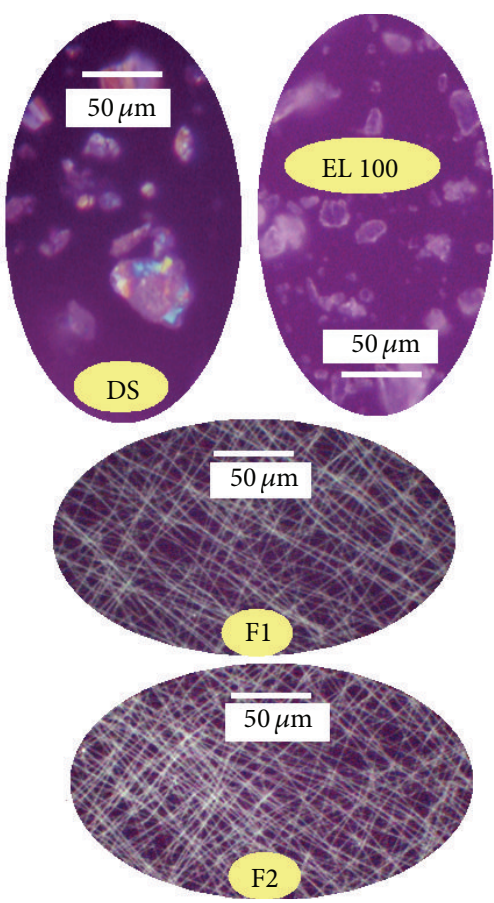<smiles>O=C(Cc1ccccc1Nc1c(Cl)cccc1Cl)O[O-]</smiles>

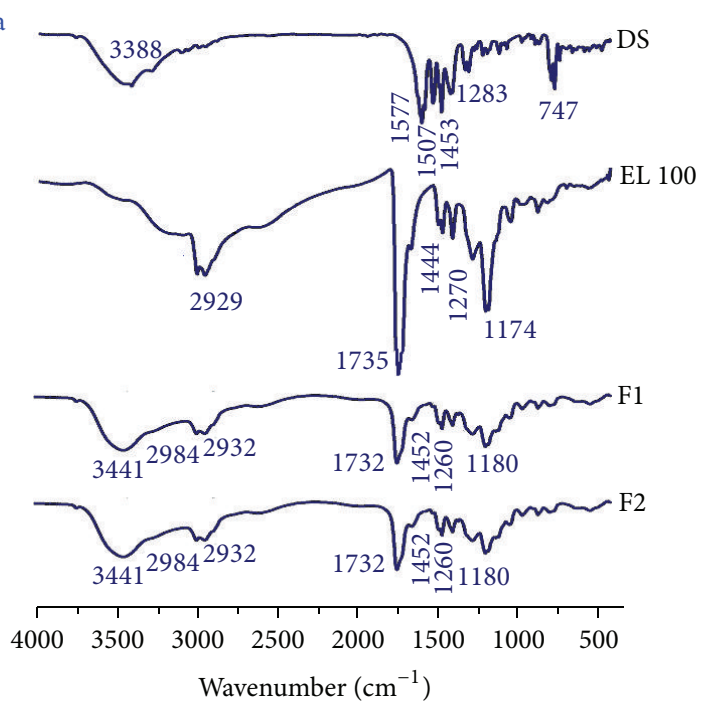

(b)

FIGURE 5: XRD patterns (a) and ATR-FTIR spectra (b) of the components and their composite nanofibers.

distance for water diffusing into the nanofibers and the drug diffusing into the dissolution medium. Showed in Figure 6(b) is a schematic diagram of the drug DS to explain how the drug goes into the dissolution medium from the nanofibers. Although F1 had a larger width than F2, the drug went into the medium always by the shortest way. The thickness of the flat nanofibers F1 was the determined factor to control the drug release. Because of a flat morphology, the longest diffusion distance $\left(R_{1}, 280 \mathrm{~nm}\right.$ by estimate from their FESEM images) of the nanofibers F1 was smaller than the diameters of F2
$\left(R_{2}=D_{2} / 2\right)$ and thus exhibited a poorer colon-targeted sustained drug release profile.

To further investigate the drug controlled release mechanism, the drug release profiles from the nanofibers F1 and F2 were analyzed using the Peppas equation [22]:

$$
Q=k t^{n},
$$

where $Q$ is the drug release percentage, $t$ is the release time, $k$ is a constant reflecting the structural and geometric characteristics of the fibers, and $n$ is an exponent that indicates 


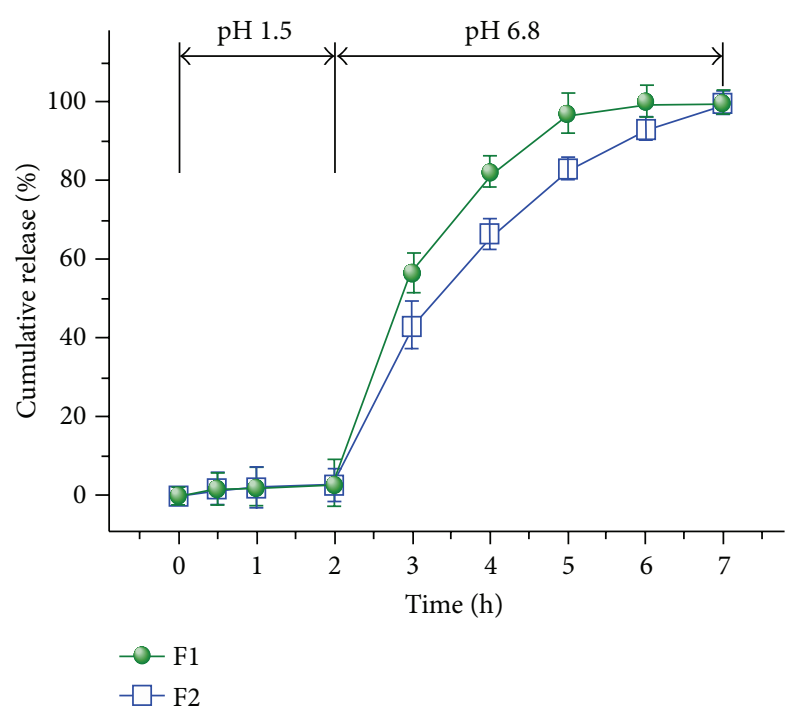

(a)

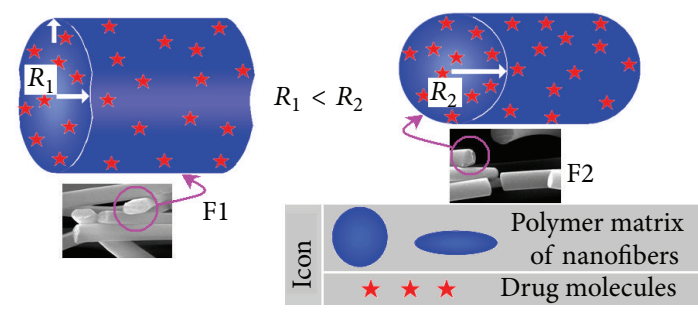

(b)

FIgURE 6: In vitro drug dissolution profiles $(n=6)$ (a) and a schematic diagram of the drug release distance (b).

the drug release mechanism. The results for nanofibers $\mathrm{F} 1$ and F2 yield $Q_{1}=15.78 t_{1}^{0.98}\left(R_{1}^{2}=0.9771\right)$ and $Q_{2}=19.13 t_{2}^{0.84}$ $\left(R_{2}^{2}=0.9845\right)$, respectively. Exponent values of 0.98 and 0.84 (a value of $n$ between 0.5 and 1 ) indicate that the drug releases from F1 and F2 were similarly controlled via a nonFickian diffusion mechanism; that is, the drug release from the nanofibers was controlled by a combination of diffusion and erosion mechanisms. At a pH of 6.8, both EL100 and DS are soluble. However, their dissolution behaviors are different. The dissolution polymer always takes relatively longer time because they usually involve the processes of absorption of water, swelling, and disentanglement before they are free in the dissolution media. However, DS dissolves more quickly than EL 100 as a little chemical molecule. It can dissolve with EL100 synchronously via erosion mechanism. It can also enter into the dissolution media through channels that would develop during the dissolution process along the nanofibers from the surface to the inside of the fiber, that is, via a diffusion mechanism. There is no difference of drug controlled release mechanisms, which means that it is the nanofibers' shapes that produced the different colon-targeted sustained release profiles between nanofibers F1 and F2.

\section{Conclusion}

A modified coaxial electrospinning process in which only an unspinnable mixed solvent system was used as a sheath fluid has been successfully developed to produce medicated EL100 nanofibers. FESEM observations revealed that the modified coaxial electrospinning process is an effective method for manipulating the nanofibers' shape and structural uniformity. The use of the surrounding solvents facilitated the drawing of the core DS/EL100 codissolving fluid jet, producing round composite fibers with a finer average size of $650 \pm$ $130 \mathrm{~nm}$ under a sheath-to-core flow rate ratio of 0.1 , whereas the fibers from the one-fluid electrospinning process had a flat morphology with an average width of $1280 \pm 330 \mathrm{~nm}$. However, both fiber types were similar in that the drug DS dispersed in an amorphous state in the filament-forming matrix EL100 and could be freed in a sustained manner through a combination of erosion and diffusion mechanisms in a neutral condition. Nonetheless, the fibers from the modified process exhibited a better colon-targeted sustained release performance than those from the single fluid electrospinning process in terms of release time period. The modified coaxial electrospinning process reported herein clearly extends the capability of electrospinning to fabricate functional polymer fibers with better structure and morphology and enhanced functional performance.

\section{Conflict of Interests}

The authors declare that there is no conflict of interests regarding the publication of this paper.

\section{Acknowledgments}

This work was supported by the National Science Foundation of China (nos. 51373101 and 51373100), the Natural Science Foundation of Shanghai (no. 13ZR1428900), the Key Project of the Shanghai Municipal Education Commission (no. 13ZZ113), and the Innovation Project of the College Student Fund Committee (no. SH2013167).

\section{References}

[1] S. Mitragotri and J. Lahann, "Physical approaches to biomaterial design,” Nature Materials, vol. 8, no. 1, pp. 15-23, 2009.

[2] T. Lin and X. Wang, Encyclopedia of Nanoscience and Nanotechnology, Edited by H. S. Nalwa, American Scientific, Los Angeles, Calif, USA, 2nd edition, 2011.

[3] N. Vitchuli, Q. Shi, J. Nowak et al., "Atmospheric plasma application to improve adhesion of electrospun nanofibers onto protective fabric," Journal of Adhesion Science and Technology, vol. 27, no. 8, pp. 924-938, 2013.

[4] J. Fang, H. Niu, H. Wang, X. Wang, and T. Lin, "Enhanced mechanical energy harvesting using needleless electrospun poly(vinylidene fluoride) nanofibre web," Energy \& Environmental Science, vol. 6, no. 7, pp. 2196-2202, 2013.

[5] J. Fang, X. Wang, and T. Lin, "Nanofibrous $p-n$ junction and its rectifying characteristics," Journal of Nanomaterials, vol. 2013, Article ID 758395, 7 pages, 2013.

[6] Y. Li, B. Guo, L. Ji et al., "Structure control and performance improvement of carbon nanofibers containing a dispersion of 
silicon nanoparticles for energy storage," Carbon, vol. 51, pp. 185-194, 2013.

[7] X.-Y. Li, X. Wang, D.-G. Yu et al., "Electrospun borneol-PVP nanocomposites," Journal of Nanomaterials, vol. 2012, Article ID 731382, 8 pages, 2012.

[8] D.-G. Yu, W. Chian, X. Wang, X.-Y. Li, Y. Li, and Y.-Z. Liao, "Linear drug release membrane prepared by a modified coaxial electrospinning process," Journal of Membrane Sciences, vol. 428, no. 2, pp. 150-156, 2013.

[9] D.-G. Yu, X.-Y. Li, X. Wang, W. Chian, Y.-Z. Liao, and Y. Li, "Zero-order drug release cellulose acetate nanofibers prepared using coaxial electrospinning," Cellulose, vol. 20, no. 1, pp. 379389, 2013.

[10] D. G. Yu, X. Wang, X. Y. Li, W. Chian, Y. Li, and Y. Z. Liao, "Electrospun biphasic drug release polyvinylpyrrolidone/ethyl cellulose core/shell nanofibers," Acta Biomaterialia, vol. 9, no. 3, pp. 5665-5672, 2013.

[11] D.-G. Yu, G. R. Williams, X.. Wang, X. K. Liu, H. L. Li, and S. W. A. Bligh, "Coaxial electrospinning using a concentric Teflon spinneret to prepare biphasic-release nanofibers of helicid," $R S C$ Advances, vol. 3, no. 39, pp. 17775-17783, 2013.

[12] K.-H. Roh, D. C. Martin, and J. Lahann, "Biphasic Janus particles with nanoscale anisotropy," Nature Materials, vol. 4, no. 10, pp. 759-763, 2005.

[13] S. Agarwal, A. Greiner, and J. H. Wendorff, "Functional materials by electrospinning of polymers," Progress in Polymer Science, vol. 38, no. 6, pp. 963-991, 2013.

[14] C. J. Luo, S. D. Stoyanov, E. Stride, E. Pelan, and M. Edirisinghe, "Electrospinning versus fibre production methods: from specifics to technological convergence," Chemistry Society Reviews, vol. 41, no. 13, pp. 4708-4735, 2012.

[15] A. K. Moghe and B. S. Gupta, "Co-axial electrospinning for nanofiber structures: preparation and applications," Polymer Reviews, vol. 48, no. 2, pp. 353-377, 2008.

[16] D.-G. Yu, L.-M. Zhu, C. Branford-White, S. W. A. Bligh, and K. N. White, "Coaxial electrospinning with organic solvent for controlling the size of self-assembled nanoparticles," Chemical Communications, vol. 47, no. 4, pp. 1216-1218, 2011.

[17] D.-G. Yu, C. J. Branford-White, N. P. Chatterton et al., "Electrospinning of concentrated polymer solutions," Macromolecules, vol. 43, no. 24, pp. 10743-10746, 2010.

[18] D.-G. Yu, C. Branford-White, S. W. A. Bligh, K. White, N. P. Chatterton, and L.-M. Zhu, "Improving polymer nanofiber quality using a modified co-axial electrospinning process," Macromolecular Rapid Communications, vol. 32, no. 9-10, pp. 744-750, 2011.

[19] D.-G. Yu, G. R. Williams, X. Wang, X.-K. Liu, H.-L. Li, and S. W. A. Bligh, "Dual drug release nanocomposites prepared using a combination of electrospraying and electrospinning," RSC Advances, vol. 3, no. 14, pp. 4652-4658, 2013.

[20] X.-X. Shen, D.-G. Yu, L.-M. Zhu, C. Branford-White, K. White, and N. P. Chatterton, "Electrospun diclofenac sodium loaded Eudragit L 100-55 nanofibers for colon-targeted drug delivery," International Journal of Pharmaceutics, vol. 408, no. 1-2, pp. 200-207, 2011.

[21] D.-G. Yu, J.-H. Yu, L. Chen, G. R. Williams, and X. Wang, "Modified coaxial electrospinning for the preparation of high-quality ketoprofen-loaded cellulose acetate nanofibers," Carbohydrate Polymers, vol. 90, no. 2, pp. 1016-1023, 2012.

[22] N. A. Peppas, "Analysis of Fickian and non-Fickian drug release from polymers," Pharmaceutica Acta Helvetiae, vol. 60, no. 4, pp. 110-111, 1985. 

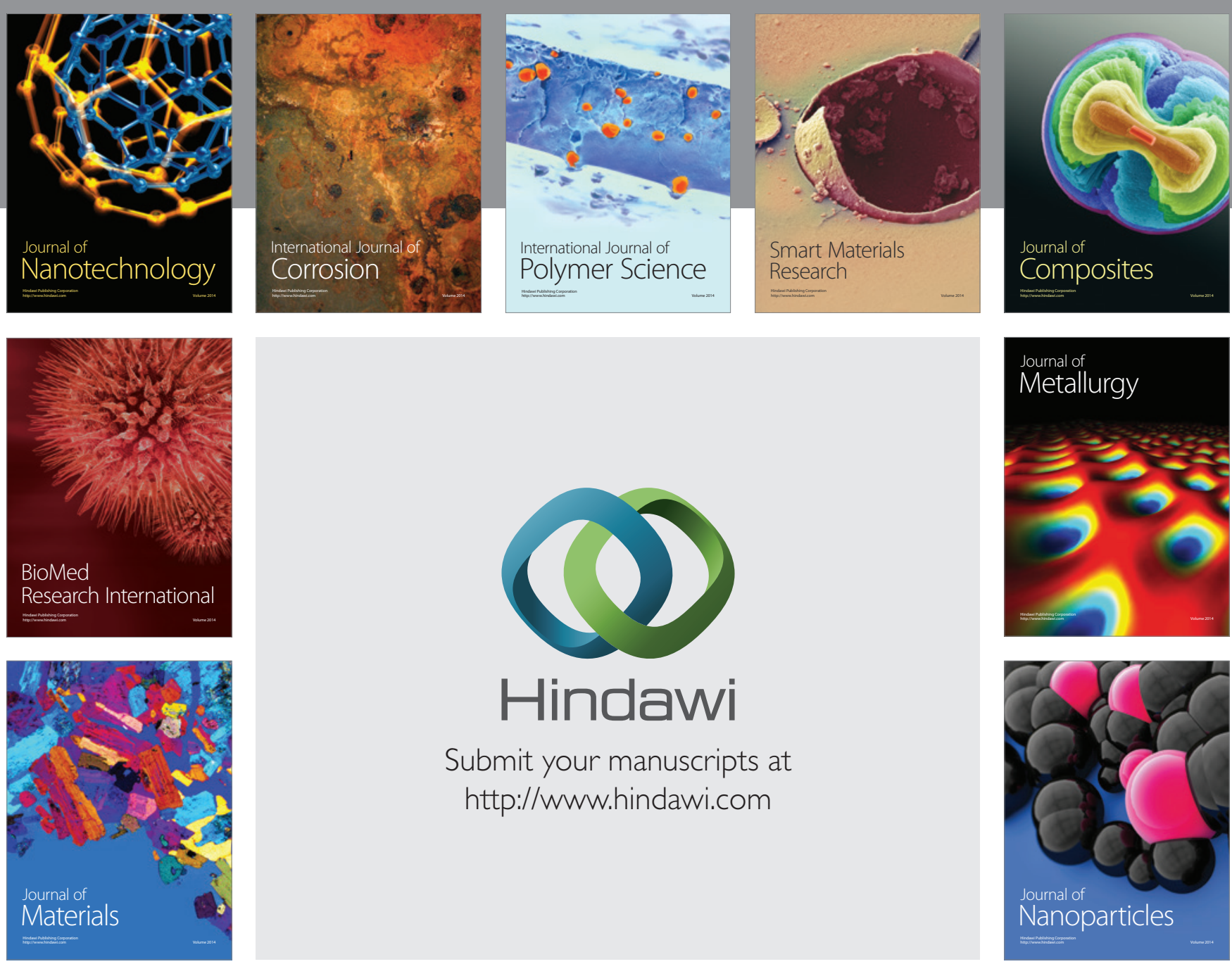

Submit your manuscripts at http://www.hindawi.com
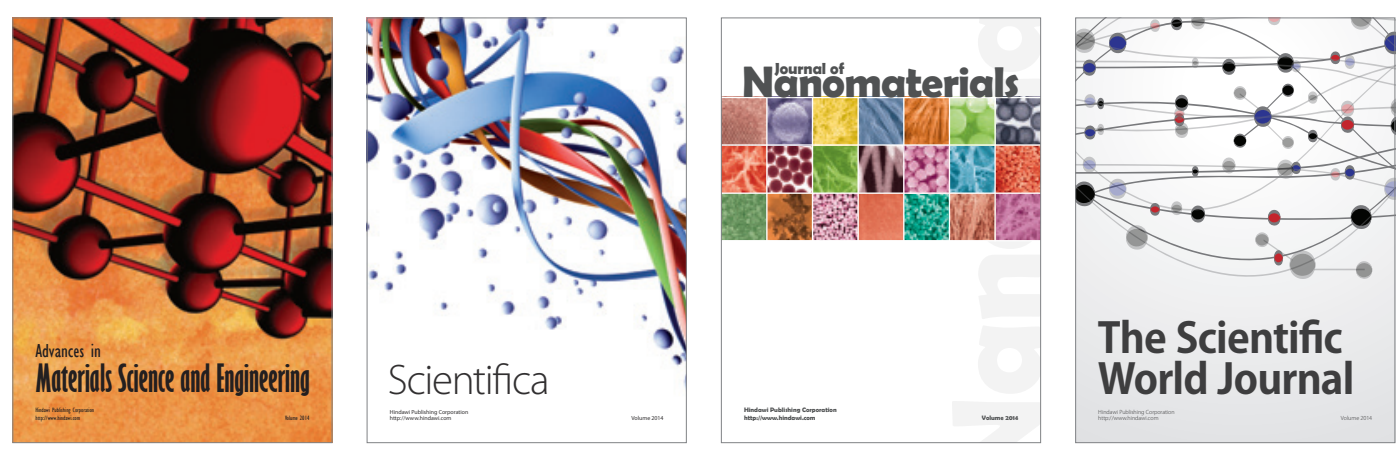

\section{The Scientific World Journal}
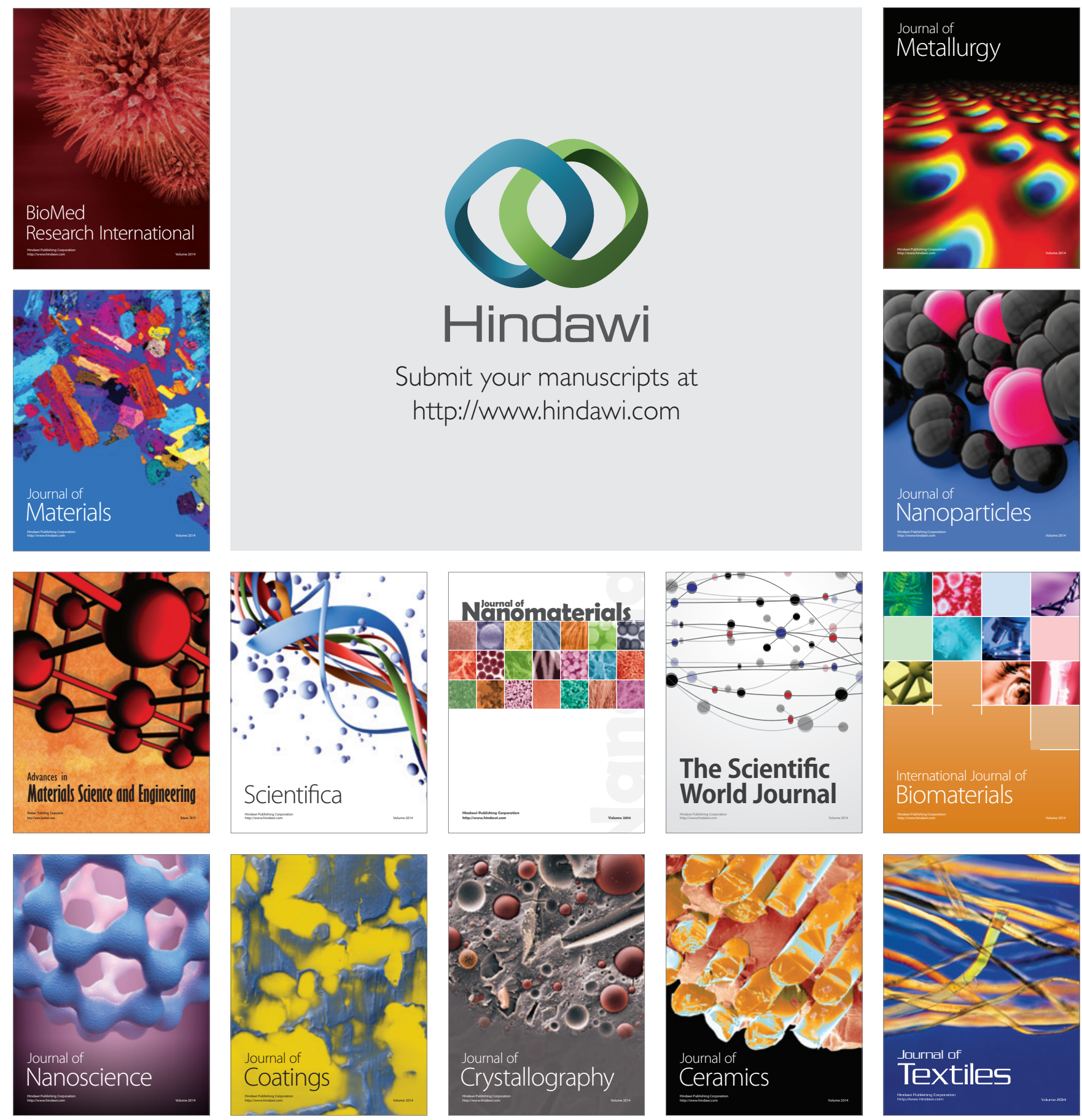